\title{
Refractive amblyopia among children aged 4-12 years in a hospital-based setting in Gaza Strip, Palestine
}

\author{
Asmaa H. Eslayeh ${ }^{1}$, Rokiah Omar ${ }^{1,2}$ and Norliza Md Fadzil ${ }^{1}$ \\ ${ }^{1}$ Optometry and Vision Science Program, Faculty of Health Sciences, Universiti Kebangsaan Malaysia, Kuala Lumpur, Malaysia \\ ${ }^{2}$ Institute of Community Ophthalmology, University of Chittagong, Chittagong Eye Infirmary and Training Complex, Chittagong, \\ Bangladesh
}

\begin{abstract}
Background: Refractive amblyopia is the most common cause of amblyopia in the Gaza Strip. However, the pattern of this condition has not yet been studied in this region. This study aimed to determine the pattern of refractive amblyopia in Gazan children aged 4-12 years.

Methods: This was a hospital-based cross-sectional study using a purposive sampling method. Children aged 4-12 years who attended the Children's Unit at Gaza Ophthalmic Hospital, Gaza Strip, Palestine from September 2019 to July 2020, were examined. A comprehensive eye test was conducted for all participants. Those who failed the eye examinations and were diagnosed with refractive amblyopia were included in the study. Demographic data and amblyopic refractive error patterns were analyzed accordingly.

Results: Of the 107 children, $72.9 \%$ were newly diagnosed with refractive amblyopia. The mean \pm standard deviation (SD) of age of the children who participated was $7.85 \pm 1.55$ years. Approximately two-thirds of the patients were female (57.9\%). Unilateral amblyopia was predominant in $60.7 \%$ of the cases. Moderate amblyopia was common (81.9\%). A total of 149 amblyopic eyes were examined in total, with a mean \pm SD (range) of best-corrected distance visual acuity and spherical equivalent of $0.45 \pm 0.19$ (0.2 to 1.3) logarithm of the minimum angle of resolution and $+0.76 \pm 4.51$ diopters $(-10.25$ to +11.50$)$. Astigmatism was the most common amblyogenic factor $(53.7 \%)$ among children with amblyopia.

Conclusions: The frequency of refractive amblyopia was $72.9 \%$, and meridional amblyopia accounted for the highest percentage. Girls were more commonly affected than boys. The majority were in the 7-year-old age group. Most cases were unilateral with moderate refractive amblyopia. Our study yields insights into the patterns of refractive amblyopia among children in the Gaza Strip.
\end{abstract}

\section{KEY WORDS}

refractive error, children, refractive amblyopia, Gaza Strip, stigmatism, best-corrected visual acuity, spherical equivalent

\section{INTRODUCTION}

Amblyopia is a unilateral or bilateral visual impairment among children. It has a good prognosisifidentified early and if appropriately treated throughout the critical period of visual development. It is caused by amblyogenic factors, such as refractive errors, strabismus, a combination of refractive error and strabismus, or vision deprivation [1].

Correspondence: Rokiah Omar, Professor of Optometry and Vision Science Program, Faculty of Health Sciences, Universiti Kebangsaan Malaysia, Kuala Lumpur, Malaysia. Email: r_omar@ukm.edu.my. ORCHID iD: https://orcid.org/0000-0001-9485-1620

How to cite this article: Eslayeh AH, Omar R, Fadzil NM. Refractive amblyopia among children aged 4-12 years in a hospital-based setting in Gaza Strip, Palestine. Med Hypothesis Discov Innov Ophthalmol. 2021 Fall; 10(3): 107-113. https://doi.org/10.51329/mehdiophthal1428

Received: 03 August 2021; Accepted: 02 November 2021

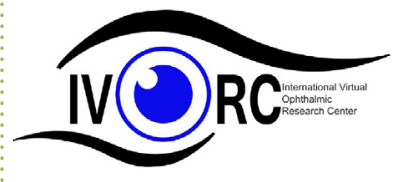

Copyright (C) Author(s). This is an open-access article distributed under the terms of the Creative Commons Attribution-NonCommercial 4.0 International License (http://creativecommons.org/licenses/by-nc/4.0/) which permits copy and redistribute the material just in noncommercial usages, provided the original work is properly cited. (c) (i) (s) 
Refractive amblyopia can be isometropia or anisometropia. Anisometropic amblyopia is the most common cause of amblyopia [2]. Anisometropic amblyopia is unlikely to be identified by parents, as the children do not complain of blurred vision and the eye appears normal, with no eye deviation as seen in strabismic amblyopia [2-4]. Anisometropic amblyopia occurs when there is a difference in the refractive error between the eyes. Isoametropic amblyopia develops when there is a bilateral reduction in visual acuity, normally resulting from an uncorrected refractive error that is equally high in both eyes $[1,2,4]$. The efficacy of amblyopic treatment is influenced by age; therefore, early detection is recommended, and vision screening has proven to be an effective method in this respect $[5,6]$.

In an 8-year review of the records of 33,183 patients, in the age range of 0-80 years, from Hebron, Palestine, refractive error was the most common eye disease, occurring in $26 \%$ of individuals. This condition was statistically significantly more frequent among the 5-9- and 10-19-year age group. Strabismus and amblyopia accounted for $6 \%$ of cases, which were more common among children aged 5-9 years [7]. In a cross-sectional study on vision screening programs for Palestinian preschool children aged 3-5 years in Nablus city, both anisometropia and significant refractive errors were the main causes of amblyopia [8]. Awad et al. reported amblyopia as the most common cause of low vision in children less than 6 years old [9]. These findings underline the importance of an in-depth investigation to understand the pattern of amblyopia and low vision in children in this region, as it would allow an intervention program to be proposed and implemented.

Despite the current understanding, vision screening in the Gaza Strip is carried out in school only at the age of 7 years, i.e., at Standard 1, which is equivalent to Grade 2 in the US school system. There is no vision screening program for preschool children in this region. This leads to late detection, and consequently, poor prognosis for amblyopia cases among Gazan children. Hence, a comprehensive study is required to improve understanding of the pattern of refractive amblyopia among Gazan children. Therefore, this study aimed to determine the refractive amblyopia pattern at a government hospital among Gazan children aged between 4-12 years old. Suggestions on a comprehensive approach to refractive amblyopia treatment as part of the eye and vision care services in the Gaza strip can occur when the refractive amblyopia pattern among Gazans is understood. Furthermore, a reliable and affordable treatment for this condition can ensure that Gazan children have better vision to facilitate their capacity for learning and performing daily activities.

\section{METHODS}

This cross-sectional study was conducted from September 2019 to July2020, within COVID-19 standard operating procedures, at Gaza Ophthalmic Hospital, Gaza Strip, Palestine. The study was conducted in accordance with the tenets of the Declaration of Helsinki. The Department of Health Research and Human Resource Development, Ministry of Health, Gaza Strip, Palestine, and Palestinian Health Research Council provided approval for this study (Number: PHRC/HC/655/). The Secretariat for Research and Ethics, Universiti Kebangsaan Malaysia, approved the study with REF NO: PPI/111/8/JEP-2020-183. The protocols of the study were explained to the parents, and informed consent was obtained before enrolment of the children in the study. Participation by the children was on a volunteer basis. The inclusion criteria were an age between 4 and 12 years and a diagnosis of refractive amblyopia. Uncooperative children, children who had previously undergone surgery, or who had any ocular pathologies, were excluded from the study. The sampling method was purposive.

All the participants underwent comprehensive eye tests and examinations. At the first visit, the children underwent distance visual acuity test using the Snellen illiterate E chart, auto-refractometer test (Auto Ref/ Keratometer ARK-1a; Nidek Co., Ltd., Gamagori, Aichi, Japan), squint assessment, and cycloplegic refraction by optometrists. Thereafter, an ophthalmologist performed a comprehensive ocular examination. One week later, a post-mydriatic test was conducted, and spectacles were prescribed to the children as needed. After 4 weeks of spectacle adaptation, visual acuity was reviewed at the third visit. If the child still had an absolute reduction in visual acuity $\geq 6 / 9$ in either one or both eyes, the child was diagnosed with refractive amblyopia. Information was collected and tabulated for this study. Subsequently, the child was referred for appropriate patching treatment by an optometrist; a description of this treatment is beyond the scope of this study.

The severity of amblyopia was defined based on the protocol of Amblyopia Treatment Studies (ATSs), implemented by the Pediatric Eye Disease Investigator Group (PEDIG) [10,11] as follows: mild amblyopia is $6 / 7.5(20 / 25)$ to $6 / 9(20 / 30)$, moderate amblyopia is $6 / 12(20 / 40)$ to $6 / 24(20 / 80)$, and the severe amblyopia is $6 / 30(20 / 100)$ to $6 / 120(20 / 400)$. Anisometropic amblyogenic factors were set as hyperopia $>1.00 \mathrm{D}$, myopia $>3.00 \mathrm{D}$ and astigmatism $>1.50 \mathrm{D}$, while isometropic amblyogenic factors were hyperopia $>5.00 \mathrm{D}$, myopia > $8.00 \mathrm{D}$, and astigmatism $>2.50 \mathrm{D}$ [12]. Data collected included patient demographics and all eye test findings.

Statistical analysis was performed using IBM SPSS Statistics for Windows (version 22.0; IBM Corp., Armonk, 
NY, USA). Statistical analysis was done in two parts, namely descriptive statistics and inferential statistics. For the descriptive statistics, demographic data for age, and gender along with the clinical profile were reported. In addition, the mean, and standard deviation (SD) values were also reported, where applicable. This is presented in the form of tables. The Shapiro-Wilk test for normality were adopted. Statistical significance was set at $P<0.05$.

\section{RESULTS}

A total of 107 children (149 amblyopic eyes) fulfilling the inclusion criteria were enrolled. Table 1 presents the demographic information of the children. Data were normally distributed, and a parametric test was used for further analysis. The mean \pm SD (range) of age group of the amblyopic children was $7.85 \pm 1.55$ years old (4-12 years old). The majority (41.1\%) of them were aged 7 years. Of the 107 children who participated, 62 (57.9\%) were girls, and the remainder was boys. Of the 107 children, $72.9 \%$ were new amblyopic cases who had not received any prior treatment, and needed prescription spectacles. The remainder had prescription spectacles and underwent patching treatment for the first time.

Unilateral amblyopia was more common than bilateral amblyopia (65 cases, $60.7 \%$ versus 42 cases, $39.3 \%$ ). Children with bilateral amblyopia were significantly younger, with a mean \pm SD of age of $7.1 \pm 1.4$ years, than unilateral cases, with a mean \pm SD of age of $7.9 \pm 1.7$ years $(P<0.05)$. Among the patients with unilateral amblyopia, amblyopia was more common in the left eye (38 cases, 58.5\%). Anisometropic amblyopia was present in $62.2 \%$ of the cases. The mean \pm SD (range) of uncorrected distance visual acuity (UCDVA) was $0.73 \pm 0.27 \log$ MAR (0.3 to $1.5 \log \mathrm{MAR}$ ), while the mean $\pm \mathrm{SD}$ (range) of best-corrected distance visual acuity (BCDVA) was $0.45 \pm 0.19$ logMAR (0.2 to $1.3 \log$ MAR). Demographic information, UCDVA, and baseline BCDVA are shown in Table 1.

Table 2 shows the amblyopic severity levels in 149 eyes according to the BCDVA achieved by the participants. Moderate amblyopia was the most common (81.9\%), followed by severe amblyopia (13.4\%) and mild amblyopia (4.7\%). As our study focused only on refractive amblyopia, we found three amblyogenic factors (Table 3 ). The most common factor was astigmatism $(53.7 \%)$, followed by hyperopia $(30.9 \%)$, and myopia (15.4\%). Table 3 shows the mean \pm SD of the dioptric power by refractive error type.

According to the axis, among the astigmatic eyes (80 eyes), with-the-rule astigmatism (WTR) was the most

Table 1. Demographic characteristics and visual information of the refractive amblyopic children aged 4-12 years in Gaza strip

\begin{tabular}{|c|c|c|c|c|c|c|}
\hline Category & \multicolumn{2}{|c|}{ Description } & \multicolumn{4}{|l|}{ n (\%) } \\
\hline \multirow[t]{9}{*}{ Age $(y), n=107$} & \multicolumn{2}{|l|}{4} & \multicolumn{4}{|l|}{$2(1.9)$} \\
\hline & \multicolumn{2}{|l|}{5} & \multicolumn{4}{|l|}{$6(5.6)$} \\
\hline & \multicolumn{2}{|l|}{6} & \multicolumn{4}{|l|}{$14(13.1)$} \\
\hline & \multicolumn{2}{|l|}{7} & \multicolumn{4}{|l|}{$44(41.1)$} \\
\hline & \multicolumn{2}{|l|}{8} & \multicolumn{4}{|l|}{$14(13.1)$} \\
\hline & \multicolumn{2}{|l|}{9} & \multicolumn{4}{|l|}{$11(10.3)$} \\
\hline & \multicolumn{2}{|l|}{10} & \multicolumn{4}{|l|}{$11(10.3)$} \\
\hline & \multicolumn{2}{|l|}{11} & \multicolumn{4}{|l|}{$2(1.9)$} \\
\hline & \multicolumn{2}{|l|}{12} & \multicolumn{4}{|l|}{$3(2.8)$} \\
\hline Gender, $\mathbf{n}=107$ & \multicolumn{2}{|c|}{ Male / Female } & \multicolumn{4}{|c|}{$45(42.1) / 62(57.9)$} \\
\hline Amblyopia, $\mathbf{n}=107$ & \multicolumn{2}{|c|}{ Unilateral / Bilateral } & \multicolumn{4}{|c|}{$65(60.7) / 42(39.3)$} \\
\hline \multirow{15}{*}{$\begin{array}{l}\text { Visual acuity } \\
n=149 \text { eyes }\end{array}$} & \multirow[t]{2}{*}{$\log M A R$} & Meter & \multicolumn{2}{|c|}{ OD, $n=69$} & \multicolumn{2}{|l|}{ OS, $\mathbf{n}=\mathbf{8 0}$} \\
\hline & & & $\begin{array}{l}\text { UCDVA } \\
\text { n }(\%)\end{array}$ & $\begin{array}{l}\text { BCDVA } \\
\text { n }(\%)\end{array}$ & $\begin{array}{l}\text { UCDVA } \\
\mathbf{n}(\%)\end{array}$ & $\begin{array}{l}\text { BCDVA } \\
\text { n }(\%)\end{array}$ \\
\hline & 1.3 & $6 / 120$ & $6(8.7)$ & $1(1.4)$ & $6(7.5)$ & $0(0.0)$ \\
\hline & 1.2 & $6 / 95$ & $0(0.0)$ & $0(0.0)$ & $2(2.5)$ & $0(0.0)$ \\
\hline & 1.1 & $6 / 75$ & $5(7.2)$ & $0(0.0)$ & $1(1.3)$ & $0(0.0)$ \\
\hline & 1.0 & $6 / 60$ & $8(11.6)$ & $2(3.0)$ & $13(16.3)$ & $1(1.3)$ \\
\hline & 0.9 & $6 / 48$ & $0(0.0)$ & $0(0.0)$ & $0(0.0)$ & $0(0.0)$ \\
\hline & 0.8 & $6 / 38$ & $4(5.8)$ & $3(4.3)$ & $10(12.5)$ & $10(12.5)$ \\
\hline & 0.7 & $6 / 30$ & $8(11.6)$ & $3(4.3)$ & $9(11.2)$ & $1(1.3)$ \\
\hline & 0.6 & $6 / 24$ & $14(20.3)$ & $4(5.8)$ & $17(21.2)$ & $10(12.5)$ \\
\hline & 0.5 & $6 / 19$ & $18(26.1)$ & $13(18.8)$ & $14(17.5)$ & $14(17.5)$ \\
\hline & 0.4 & $6 / 15$ & $4(5.8)$ & $10(14.5)$ & $5(6.2)$ & $11(13.8)$ \\
\hline & 0.3 & $6 / 12$ & $2(2.9)$ & $30(43.5)$ & $3(3.8)$ & $29(36.2)$ \\
\hline & 0.2 & $6 / 9.5$ & $0(0.0)$ & $3(4.3)$ & $0(0.0)$ & $4(5.0)$ \\
\hline & 0.1 & $6 / 7.5$ & $0(0.0)$ & $0(0.00)$ & $0(0.0)$ & $0(0.0)$ \\
\hline
\end{tabular}

Abbreviations: y, years; n, number; \%, percentage; OD, right eye; OS, left eye; logMAR, logarithm of the minimum angle of resolution; UCDVA, uncorrected distance visual acuity; BCDVA, best-corrected distance visual acuity. 
Table 2. Distribution of level of amblyopic severity according to eyes with best-corrected distance visual acuity among 4-12 yearsold children in the Gaza Strip

\begin{tabular}{|c|c|c|c|}
\hline \multicolumn{2}{|c|}{ BCDVA } & \multirow{2}{*}{$\begin{array}{l}n=149 \text { eyes } \\
\text { n (\%) }\end{array}$} & \multirow{2}{*}{$\begin{array}{l}\text { Severity of Amblyopia } \\
\text { n (\%) }\end{array}$} \\
\hline logMAR & Meter & & \\
\hline 1.3 & $6 / 120$ & $1(0.7)$ & \multirow[t]{7}{*}{ Severe amblyopia } \\
\hline 1.2 & $6 / 95$ & $0(0.0)$ & \\
\hline 1.1 & $6 / 75$ & $0(0.0)$ & \\
\hline 1.0 & $6 / 60$ & $3(2.0)$ & \\
\hline 0.9 & $6 / 48$ & $0(0.0)$ & \\
\hline 0.8 & $6 / 38$ & $12(8.0)$ & \\
\hline 0.7 & $6 / 30$ & $4(2.7)$ & \\
\hline 0.6 & $6 / 24$ & $14(9.4)$ & \multirow[t]{4}{*}{ Moderate amblyopia } \\
\hline 0.5 & $6 / 19$ & $28(18.8)$ & \\
\hline 0.4 & $6 / 15$ & $21(14.1)$ & \\
\hline 0.3 & $6 / 12$ & $59(39.6)$ & \\
\hline 0.2 & $6 / 9.5$ & $7(4.7)$ & \multirow{2}{*}{$\begin{array}{l}\text { Mild amblyopia } \\
7(4.7 \%)\end{array}$} \\
\hline 0.1 & $6 / 7.5$ & $0(0.0)$ & \\
\hline
\end{tabular}

Abbreviations: n, number; \%, percentage; logMAR, logarithm of the minimum angle of resolution; BCDVA,

best-corrected distance visual acuity.

Table 3. Level of refractive errors severity according to eyes and dioptric power of the children

\begin{tabular}{|c|c|c|c|}
\hline Type of refractive error, $n$ (\%) & Level of severity & $\begin{array}{l}\text { Eyes } \\
\text { n }(\%)\end{array}$ & $\begin{array}{l}\text { Power }(\text { D) } \\
\text { Mean } \pm \text { SD }\end{array}$ \\
\hline \multirow[t]{4}{*}{$\begin{array}{l}\text { Astigmatism } \\
80(53.7)\end{array}$} & $\begin{array}{l}\text { Low Astigmatism } \\
(-0.50 \mathrm{D} \text { to }-1.00 \mathrm{D})\end{array}$ & $0(0.0)$ & - \\
\hline & $\begin{array}{l}\text { Moderate Astigmatism } \\
(-1.25 \mathrm{D} \text { to }-2.50 \mathrm{D})\end{array}$ & $19(23.7)$ & $-2.30 \pm 0.37$ \\
\hline & $\begin{array}{l}\text { High Astigmatism } \\
(-2.75 \mathrm{D} \text { to }-5.00 \mathrm{D})\end{array}$ & $61(76.3)$ & $-3.65 \pm 0.62$ \\
\hline & Total & $80(100)$ & $-3.33 \pm 0.81$ \\
\hline \multirow[t]{4}{*}{$\begin{array}{l}\text { Hypermetropia } \\
46(30.9)\end{array}$} & $\begin{array}{l}\text { Low Hyperopia } \\
(+0.50 \mathrm{D} \text { to }+2.99 \mathrm{D})\end{array}$ & $4(8.7)$ & $+2.03 \pm 0.65$ \\
\hline & $\begin{array}{l}\text { Moderate Hyperopia } \\
(+3.0 \mathrm{D} \text { to }<+6.0 \mathrm{D})\end{array}$ & $27(58.7)$ & $+4.32 \pm 0.80$ \\
\hline & $\begin{array}{l}\text { High Hyperopia } \\
(\geq+6.0 \mathrm{D})\end{array}$ & $15(32.6)$ & $+7.34 \pm 1.75$ \\
\hline & Total & $46(100)$ & $+5.12 \pm 2.05$ \\
\hline \multirow[t]{4}{*}{$\begin{array}{l}\text { Myopia } \\
23(15.4)\end{array}$} & $\begin{array}{l}\text { Low Myopia } \\
(-0.50 \mathrm{D} \text { to }-2.99 \mathrm{D})\end{array}$ & $3(13)$ & $-2.10 \pm 0.14$ \\
\hline & $\begin{array}{l}\text { Moderate Myopia } \\
(-3.00 \mathrm{D} \text { to }-5.99 \mathrm{D})\end{array}$ & $7(30.4)$ & $-4.18 \pm 0.77$ \\
\hline & $\begin{array}{l}\text { High Myopia } \\
(\geq-6.00 \mathrm{D})\end{array}$ & $13(56.5)$ & $-7.71 \pm 0.91$ \\
\hline & Total & $23(100)$ & $-5.90 \pm 2.34$ \\
\hline
\end{tabular}

Abbreviations: n, number; \%, percentage; power, dioptric power of refractive error D, diopter; SD, standard deviation.

Table 4. Spherical equivalent for right and left eyes of refractive amblyopic children

\begin{tabular}{|l|l|l|}
\hline Eye & $\mathbf{n}(\%)$ & SE (D), Mean \pm SD (Range) \\
\hline OD & $69(46.3)$ & $+0.1 \pm 4.62(-9.50$ to +11.50$)$ \\
\hline OS & $80(53.7)$ & $+1.33 \pm 4.36(-10.25$ to +11.50$)$ \\
\hline Total & $149(100)$ & $+0.76 \pm 4.51(-10.25$ to +11.50$)$ \\
\hline
\end{tabular}

Abbreviations: n, number; \%, percentage; OD, right eye; OS, left eye; SE, spherical equivalent; D, diopter; SD, standard deviation.

common type (66 eyes, $82.5 \%$ ), followed by oblique astigmatism (14 eyes, 17.5\%). None of the children had against the rule astigmatism. Furthermore, according to refractive error classification, mixed astigmatism (MA) was the most common type (27 eyes, 33.8\%), followed by compound hyperopic astigmatism ( 25 eyes, $31.3 \%$ ), compound myopic astigmatism (12 eyes, $15 \%)$, simple myopic astigmatism (12 eyes, $15 \%)$, and simple hyperopic astigmatism (4 eyes, $5 \%$ ).

Among the 149 amblyopic eyes, the mean \pm SD of spherical equivalent $(\mathrm{SE})$ was $+0.76 \pm 4.51 \mathrm{D}$. Of these, $46.3 \%$ were right eyes with the mean SD of SE of + 0.1 $\pm 4.62 \mathrm{D}$, whereas the majority were on the left eye (53.7\%) with a mean \pm SD of SE of $+1.33 \pm 4.36 \mathrm{D}$. Table 4 shows the mean \pm SD (range) of the SE of the right and left eyes. 


\section{DISCUSSION}

The frequency of refractive amblyopia in this study was 72.9\% among treatment-naïve Gazan children aged 4-12 years. These findings suggest that the majority of children who attended the clinic were newly diagnosed with refractive amblyopia. Furthermore, refractive error was a high-risk factor for amblyopic children who attended the hospital-based referral center in Gaza. Hence, good refractive services must be provided together with amblyopic therapy.

A population-based study of 226 patients with strabismus in the Gaza Strip revealed moderate and higher degree hypermetropia as the most common refractive error, mostly in esotropia. Mild WTR astigmatism was the most common type of astigmatism [13]. A retrospective review of 1,675 patients who attended the eye clinic of the Jordanian Field Hospital in the Gaza Strip over the period of 1 year revealed refractive errors as the most common ocular disease, accounting for $19 \%$ of cases. Refractive error with the need for spectacle correction was the most common presenting disorder in young patients [14]. Our study indicates a high frequency of refractive errors in this region. A hospital-based study by Banayot revealed that $26 \%$ of the cases had refractive errors. In contrast to our study, his study was an 8 years' retrospective review of 33,183 patients' records over a wide age range of 0-80 years at St John Eye Tertiary Hospital, Hebron, Palestine. Strabismus and amblyopia accounted for only $6 \%$ of cases and were more common among children aged 5-9 years [7]. These findings reinforce that refractive error is one of the risk factors for amblyopia among children in Palestine. In another hospital-based study at St. John Eye Hospital, the same author reviewed 1,102 records of patients aged < 16 years over a period of 1 year. Similar to his previous study in the same hospital, refractive error was the most common ocular disease, accounting for $31.6 \%$ of cases, while the third most common was strabismus and amblyopia (13.8\%) [15]. The mean age of children seeking eye care was slightly lower ( 7.14 years) than in our study, where the mean age was 7.85 years. In that study, the age group of 0-5 years was the largest group seeking eye care [15]. In our study, the age group of the 7-year-old was the largest. These differences are likely due to the different objectives of each study. Nevertheless, based on the previous findings and the current study, we can infer that an initial vision screening program is critical for early detection of amblyopia in the Gaza Strip region.

When considering the types of refractive amblyopia, we found that meridional amblyopia accounted for the highest percentage, followed by hyperopic amblyopia and myopic amblyopia. Among the astigmatic eyes, WTR was the most common type (82.5\%) based on the axis, while MA was the most common type (33.8\%) according to the classification of refractive errors. Similarly, Sapkota et al. reported that the majority of the participants had astigmatic amblyopia (59.2\%) [16]. However, in a school-based population study, Amer and Tarshawi discovered that manifest hypermetropia was the most common type of refractive error, followed by astigmatism, in children between 7 years old or Standard 1 equivalent to Grade 2 and 12 years old or Standard 6 equivalent to Grade 7 in the Gaza Strip. Similar to our study, the most common type of astigmatism was WTR astigmatism [17]. Another study, by Al-Haddad et al., also concluded that hyperopia was the main cause (54\%) of refractive amblyopia, followed by astigmatism and myopia. However, refractive amblyopia was more prevalent in children aged 3-7 years, while strabismic amblyopia was common in the younger age group (<3 years) [18]. In a population-based study on the prevalence of amblyopia in Mashhad, Iran, anisometropic amblyopia, and isoametropic amblyopia were the first and second most common factors, respectively. MA was the most prevalent subtype of refractive error among amblyopic children [19]. Interestingly, we found that $81.9 \%$ of the patients in our study had moderate refractive amblyopia. The UCDVA (mean \pm SD) was $0.73 \pm 0.27 \log M A R$, while the BCDVA was $0.45 \pm 0.19 \log$ MAR, which still put the children in the moderate category of refractive amblyopia following prescription of spectacles. Rajavi et al. [20] found that the majority (93.4\%) of their children also fell in the category of moderate refractive amblyopia with a BCDVA range from 0.3 to $0.7 \log$ MAR [20]. However, in Egypt, severe refractive amblyopia was reported at a higher rate $(57.1 \%)$ than mild to moderate refractive amblyopia [21].

In this study, we found that the majority of patients $(41.1 \%)$ were diagnosed with refractive amblyopia at the age of 7 years. Based on age, these children had already passed the critical period of visual development. Therefore, these children were exposed to a higher risk of treatment failure. Previous studies have recommended that patching therapy could be more effective if applied at a younger age [22-24]. In terms of laterality, in our study, unilateral amblyopia was significantly more common (65 cases, $60.7 \%$ ), particularly in the older age group, than bilateral amblyopia. The majority of unilateral amblyopic cases involved the left eye (58.5\%). Our study was in agreement with a study conducted in Iran, where $64.3 \%$ of the children had unilateral amblyopia, even though that study investigated a 7-12-year-old group. The difference between the right (56.5\%) and left eyes (43.5\%) in the prevalence of amblyopia was not significant [20]. For the refractive bilateral amblyopia cases identified among our children, spectacle treatment may yield a good prognosis, according to a previous study [25]. Our findings further suggest that the visual impairment experienced by children in the Gaza Strip may be missed or unrecognized by parents. 
Hence, most of the cases seen in our study were new, and they had not received any prior treatment.

In terms of gender, our study showed that girls (57.9\%) were affected more than boys. However, Sapkota et al. found that it was more common in boys [16]. Therefore, further investigations are required. A possible explanation may be related to cultural practice in Palestinian society, where girls seek eye care at the primary unit, as opposed to boys. However, in a multi-country refractive error study of 39,321 children aged 5-15 years, the prevalence of amblyopia was not associated with gender [26].

Refractive amblyopia patterns seem to vary between the current and previous studies [16-18, 20, 21]. These include the most common types, severity, laterality, level of vision, age, and gender distribution of refractive amblyopia in the Gaza Strip region. Hence, these findings indicate that uncorrected refractive error remains a high-risk factor for the development of refractive amblyopia in children, regardless of the pattern. Refractive amblyopia is avoidable, and an early vision screening program can alleviate this issue [27], particularly in the Gaza Strip. Possible reasons for challenges faced for the high frequency of refractive amblyopia cases among children in the Gaza Strip include that the children did not complain of blurry vision. The parents were not aware that their children had blurred vision, as refractive amblyopia signs and symptoms are not visible as are those of strabismus amblyopia, where the parents can notice the deviated eye [2-4]. Hence, a comprehensive vision screening program is recommended at preschool age or at 4-6 years of age. In collaboration with other stakeholders, the Health Ministry can implement an awareness program on uncorrected refractive error and amblyopia. Previous studies have shown the effectiveness of conducting vision screening at an early age [27-30]. Good vision will enable children to do better in school and can facilitate teaching and learning [31, 32].

Although this was a cross-sectional study, the data collected were normally distributed; therefore, the findings can be used for comparison with other hospital-based population studies. It is hoped that this study will create awareness among policymakers, particularly in the Gaza Strip, to design and implement comprehensive vision screening programs for children as young as 4 years of age. In addition, the actual prevalence and incidence estimation of Gazan children aged 4-12 years is recommended for a population-based study in future. The Amblyopia Database Registry can be established. This can form part of the Prevention of Avoidable Blindness Movement, to ensure the sustainability of the program. The Amblyopia Database can also provide information to health workers. Furthermore, educational materials on uncorrected refractive error and amblyopia can be developed and provided to the public to improve awareness. Research, development, and better treatment for amblyopic children can also be provided with information available from the Amblyopia Database.

\section{CONCLUSIONS}

The frequency of refractive amblyopia was $72.9 \%$. The predominant age group was 7 years old. Anisometropic amblyopia and moderate amblyopia were also common (62.2\% and $81.9 \%$, respectively). Astigmatism is the most common amblyogenic factor. Development of an early vision screening program is highly recommended, and should be accompanied by appropriate treatment options to be applied early, before school age. With a better understanding of refractive amblyopia patterns among the Gazan society, a comprehensive approach, particularly refractive amblyopia prevention programs, such as preschool vision screening programs and management, can be recommended as part of the eye and vision care services provided by the government hospital in the Gaza Strip.

\section{ETHICAL DECLARATIONS}

Ethical approval: The Department of Health Research and Human Resource Development, Ministry of Health, Gaza Strip, Palestine, and Palestinian Health Research Council provided approval for this study (Number: $\mathrm{PHRC} / \mathrm{HC} / 655 /)$. The Secretariat for Research and Ethics, Universiti Kebangsaan Malaysia, approved the study with REF NO: PPI/111/8/JEP-2020-183. This study also followed the tenets of the Declaration of Helsinki. The protocols of the study were explained to the parents, and informed consent was obtained before enrolment of the children in the study. Participation by the children was on a volunteer basis.

Conflict of interests: None.

FUNDING

None.

ACIKNOWLED GEMENTS

We would like to thank all participants for their support in completing this project. 


\section{REFERENCES}

1. Roda M, Pellegrini M, Di Geronimo N, Vagge A, Fresina M, Schiavi C. Binocular treatment for amblyopia: A meta-analysis of randomized clinical trials. Plos One. 2021;16(10): e0257999. doi: 10.1371/journal.pone.0257999 pmid: 34624028

2. Ikuomenisan S, Musa K, Aribaba O, Onakoya A. Prevalence and pattern of amblyopia among primary school pupils in Kosofe town, Lagos state, Nigeria. Nigerian Postgraduate Medical Journal. 2016;23(4): 196-201. doi: 10.4103/1117-1936.196261 pmid: 28000640

3. Zhu H, Pan C, Sun Q, Huang D, Fu Z, Wang J, et al. Prevalence of amblyopia and strabismus in Hani school children in rural southwest China: a cross-sectional study. BMJ Open. 2019;9(2): e025441. doi: 10.1136/bmjopen-2018-025441 pmid: 30782934

4. El-Sobky HK, Said Ahmed K-G, El-Sawy M, Sakr SI. Incidence of amblyopia in the Menoufia University outpatient clinic. Menoufia Medical Journal. 2015;28(1): 207. doi: 10.4103/1110-2098.155996

5. Donahue SP, Arthur B, Neely DE, Arnold RW, Silbert D, Ruben JB. Guidelines for automated preschool vision screening: A 10year, evidence-based update. Journal of American Association for Pediatric Ophthalmology and Strabismus. 2013;17(1):4-8. doi: 10.1016/j.jaapos.2012.09.012 pmid: 23360915

6. Mitchell DE, MacNeill K, Crowder NA, Holman K, Duffy KR. Recovery of visual functions in amblyopic animals following brief exposure to total darkness. The Journal of Physiology. 2016;594(1):149-67. doi: 10.1113/JP270981 pmid: 26449521

7. Banayot R. The profile of eye disease in palestine: An 8-year experience at St. John Eye Hospital, Hebron. Ibnosina Journal of Medicine and Biomedical Sciences. 2018;10(4): 130. doi: 10.4103/ijmbs.ijmbs_47_18

8. Al-Labadi L, Shahin R, Eperjesi F, Al-Shanty Y, Abdel Rahman Y, Shehadeh M, et al. Prevalence of visual disorders in urban Palestinian children: a cross-sectional study. The Lancet. 2018;391. doi: 10.1016/S0140-6736(18)30355-6

9. Awad KS, Aljarousha MA, Alasmar AI, Eslayeh AH, Alshaer DY, Elbanna SM. The prevalence and major causes of low vision among children in Gaza strip, Palestine. IUG Journal of Natural Studies. 2017;25(2):85-88. Link

10. Beck RW. Clinical research in pediatric ophthalmology: The Pediatric Eye Disease Investigator Group. Current Opinion in Ophthalmology. 2002;13(5):337-40. doi: 10.1097/00055735-200210000-00008 pmid: 12218466

11. Buckle M, Billington C, Shah P, Ferris JD. Treatment outcomes for amblyopia using PEDIG amblyopia protocols: a retrospective study of 877 cases. Journal of American Association for Pediatric Ophthalmology and Strabismus. 2019;23(2):98.e1-.e4. doi: 10.1016/j.jaapos.2018.12.007 pmid: 30935990

12. Rouse MW, Cooper JS, Cotter SA, Press LJ,Tannen BM (2004). 'Care of the patient with amblyopia.' Optometric Clinical Practice Guidelines. American Optometric Association. Available at: American Optometric Association (Accessed: March 21, 2021)

13. Amer A. Relative Prevalence of Various Types of Strabismus in Patients Attending NGO's Medical Centers in Gaza Strip. Science Journal of Public Health. 2014; 3(1-1):1-5. doi: 10.11648/j.sjph.s.2015030101.11

14. Droos M, Khtatbeh O. Pattern of Eye Disease in Patients Attending the Jordanian Field Hospital in Gaza Strip. Journal of the Royal Medical Services. 2013; 20 (1): 33-6. Link

15. Banayot RG. A retrospective analysis of eye conditions among children attending St. John Eye Hospital, Hebron, Palestine. BMC Research Notes. 2016;9(1): 202. doi: 10.1186/s13104-016-2011-9 pmid: 27048163

16. Sapkota K, Pirouzian A, Matta NS. Prevalence of amblyopia and patterns of refractive error in the amblyopic children of a tertiary eye care center of Nepal. Nepalese Journal of Ophthalmology. 2013;5(1):38-44. doi: 10.3126/nepjoph.v5i1.7820 pmid: 23584645

17. Amer A, Tarshawi M. Prevalence of Manifest Hypermetropia in Primary School Children of Gaza City. Science Journal of Public Health. 2013;1(3): 131-4. doi: 10.11648/j.sph.20130103.14

18. Al-Haddad C, Ismail K, Jurdi K, Keaik M. Clinical profile and treatment outcomes of amblyopia across age groups. Middle East African Journal of Ophthalmology. 2019;26(2): 71-76. doi:10.4103/meajo.MEAJO_182_17 pmid: 31543663

19. Shahpary S, Ramin S, Azimi A, Heyrani M. The prevalence of amblyopia in 7-9-year-old schoolchildren in Mashhad. Medical Hypothesis, Discovery \& Innovation in Optometry. 2021;2(1):18-23. doi: 10.51329/mehdioptometry121

20. Rajavi Z, Sabbaghi H, Baghini A, Yaseri M, Moein H, Akbarian S, et al. Prevalence of amblyopia and refractive errors among primary school children. Journal of Ophthalmic and Vision Research. 2015;10(4): 408-16. doi: 10.4103/2008-322X.176909 pmid: 27051485

21. Rashad MA, Abd Elaziz KM, Fawzy SM, Abdel latif AAM, Abdel latif MAM. Screening of Primary School Children for Amblyopia and Amblyogenic Factors in Central Cairo, Egypt. Journal of Ophthalmology. 2018;2018:1-6. doi: 10.1155/2018/8425319 pmid: 29850217

22. Holmes JM, Levi DM. Treatment of amblyopia as a function of age. Visual Neuroscience. 2018;35. doi: 10.1017/ S0952523817000220 pmid: 29905125

23. Holmes JM. Effect of Age on Response to Amblyopia Treatment in Children. Archives of Ophthalmology. 2011;129(11): 1451-7. doi 10.1001/archophthalmol.2011.179 pmid: 21746970

24. Park SH. Current Management of Childhood Amblyopia. Korean Journal of Ophthalmology. 2019;33(6): 557-568. doi: 10.3341/ kjo.2019.0061 pmid: 31833253

25. Wallace DK, Chandler DL, Beck RW, Arnold RW, Bacal DA, Birch EE, et al. Treatment of Bilateral Refractive Amblyopia in Children Three to Less Than 10 Years of Age. American Journal of Ophthalmology. 2007;144(4):487-96. doi: 10.1016/j.ajo.2007.05.040 pmid: 17707330

26. Xiao O, Morgan IG, Ellwein LB, He M. Prevalence of Amblyopia in School-Aged Children and Variations by Age, Gender, and Ethnicity in a Multi-Country Refractive Error Study. Ophthalmology. 2015;122(9):1924-31. doi: 10.1016/j.ophtha.2015.05.034 pmid: 26278861

27. Omar R, Knight VF, Zabidi AA, Saat NZ, Li TX. Effectiveness of vision screening program conducted by pre-school teachers. Malaysian Journal of Public Health Medicine. 2018;2018(SI 1):41-50. Link

28. Tan XL, Omar R, Victor FK. Knowledge Retention Level among Pre-School Teachers in Conducting Pre-School Children Vision Screening. Jurnal Sains Kesihatan Malaysia. 2019;17(SI):149-56. doi: 10.17576/jskm-2019-17

29. Omar R, Knight VF, Tan XL, Ahmad Zahidi AA, Mohd Saat NZ. Effectiveness of Vision Screening Training Module in Improving the Level of Knowledge on Vision Screening among Pre-School Teachers in Klang Valley Malaysia. Journal of Health and Translational Medicine. 2019;22(1):58-65. doi: 10.22452/jummec.vol22no1.9

30. Mathers M, Keyes M, Wright M. A review of the evidence on the effectiveness of children's vision screening. Child: Care, Health and Development. 2010;36(6):756-80. doi: 10.1111/j.1365-2214.2010.01109.x pmid: 20645997

31. Khalaj M, Zeidi IM, Gasemi MR, Keshtkar A. The effect of amblyopia on educational activities of students aged 9 - 15 . Journal of Biomedical Science and Engineering. 2011;04(07):516-21. doi: 10.4236/jbise.2011.47066

32. Webber AL. The functional impact of amblyopia. Clinical and Experimental Optometry. 2021;101(4):443-50. doi: 10.1111/ cxo. 12663 pmid: 29484704 